\title{
Human Development Index (HDI) of the maternal country of origin as a predictor of perinatal outcomes - a longitudinal study conducted in Spain
}

S. Garcia-Tizon Larroca ${ }^{1 *}$ (D) J. Arevalo-Serrano², A. Duran Vila ${ }^{1}$, M. P. Pintado Recarte ${ }^{1}$, I. Cueto Hernandez ${ }^{1}$ A. Solis Pierna', S. Lizarraga Bonelli ${ }^{1}$ and J. De Leon-Luis ${ }^{1}$

\begin{abstract}
Background: In an era of worldwide population displacement, recent studies have identified strong associations between social situations and perinatal outcomes among immigrants. Little is known about the effect of maternal social background on pregnancy outcomes. The Human Development Index (HDI) assesses the following dimensions of human development: life expectancy, education level and income. The objective of our study was to determine if maternal HDI may be used to identify women at increased odds of poor pregnancy outcomes.

Methods: We conducted a longitudinal population-based study in a tertiary centre in Madrid, Spain. The outcome variables were maternal and perinatal/antenatal mortality, preeclampsia (PE), low birth weight (LBW), gestational diabetes mellitus (GDM), preterm delivery (PTD) before 37 and 34 gestational weeks, abnormal cardiotocography (CTG) during delivery, C-section (CS) due to abnormal CTG, pH $<7.10$ at birth, Apgar at 5 min $\leq 7$, and resuscitation type $\geq 3$. We performed multivariate logistic regression analyses adjusted for potential confounding variables to evaluate the associations between maternal HDI and perinatal outcomes.

Results: In total, 38,719 singleton infants who were born in our maternity ward between 2010 and 2016 and had perinatal outcome data available were included in this study. The neonates of women from medium/low $\mathrm{HDI}$ countries had significantly lower odds of low birth weight (LBW) than their very high HDI country counterparts (OR 0.63, 95\% Cl 0.55-0.72). However, the odds of PTD before 37 gestational weeks and PE were higher in the medium/low HDI group than the very high HDI group (OR 1.26, 95\% Cl 1.04-1.53; OR 1.35, 95\% Cl 1.02-1.79, respectively). Poorer neonatal outcomes were identified in the medium/low HDI group than the very high HDI group, including greater odds of abnormal CTG, CS due to abnormal CTG and Apgar $2 \leq 7(p<0.05)$.

Conclusions: Our findings suggest that the infants of mothers from medium/low HDI had lower odds of LBW but higher odds of PTD, PE and poor neonatal outcomes. These results support the hypothesis that maternal HDI can be used to understand the impact of maternal origin on pregnancy outcomes. Further studies are needed to confirm its validity.
\end{abstract}

Keywords: Human development index, Perinatal health, Perinatal mortality, Maternal mortality, Low Birth weight, Preterm delivery, Immigrants, Socioeconomic status

\footnotetext{
* Correspondence: gineteca@gmail.com

'Department of Obstetrics and Gynaecology, Hospital General Universitario Gregorio Marañón, Universidad Complutense de Madrid, 28029 Madrid, ES, Spain

Full list of author information is available at the end of the article
} 


\section{Background}

Social determinants comprise a broad range of factors associated with health and access to care, including income inequality, social exclusion, sense of collective efficacy and social connectedness. Pregnancy has also been found to be strongly influenced by social situations. Further, in pregnancy, social deprivation has been found to be associated with poorer gestational outcomes such as preterm birth, infant mortality and growth retardation [1-3].

Certain population groups may be more likely than others to suffer from these and other health risks because of geographical, economical and educational factors [4]. Previous studies have utilized a variety of measures to assess social situations, including educational level, other socioeconomic indicators and complex scores [5].

Regarding immigrants pregnancy care perinatal and neonatal health outcomes have sometimes been found to be worse and other times been found to be better in home than host countries.

The results of previous studies on perinatal outcomes in immigrants have been heterogeneous, as their measures of derivation were dependent upon the adjustment for variables that often differ widely across the study groups; additionally, the study designs and manner in which of socioeconomic factors were integrated differed between studies. Maternal socioeconomic status in this population has been assessed in many different ways by race/ethnicity, foreign-born status, education or deprivation [6]. This vast existing body of scientific evidence has revealed the presence of an "immigrant paradox," which suggests that women of lower socioeconomic status may have better pregnancy outcomes than native women [7].

To more accurately quantify social vulnerability and its multifactorial aspects, some groups have developed different indexes that have rarely been suitable for the evaluation of pregnancy outcomes. To identify women at increased odds of poor birth outcomes, indicators of deprivation should incorporate relevant multidimensional measures.

The Human Development Index (HDI) is a summary measure of a country's average level of achievement in the following major dimensions of human development: living a long and healthy life, being knowledgeable and having a decent standard of living. Life expectancy serves as an indicator of the health dimension; standard of living is measured in terms of gross national income per capita (GNI) and education level is evaluated as the average number of years of schooling among adults aged 25 years and older and expected of number of years of schooling among children [8].
A country obtains a higher HDI score when its population has a higher life expectancy, education level, and GNI per capita; these scores are reported within the annual Human Development Report published by the United Nations Development Programme (UNDP) [9].

Life expectancy data were provided by the UN Population Division; data on the mean number of years of schooling were obtained from the UNESCO Institute of Statistics; and GNI per capita data were provided by the World Bank and International Monetary Fund.

Maternal HDI might be a useful manner in which to identify women at increased odds of poor perinatal outcomes, as it is designed to reflect basic aspects of human development that are also relevant to prenatal care.

\section{Methods}

We conducted a longitudinal cohort study including women who delivered in the Maternidad de O'Donnell de Madrid, Spain (Hospital General Universitario Gregorio Marañon de Madrid). Data on all singleton births that occurred in our centre during the period between January 2010 and December 2016 were obtained.

Data on maternal and pregnancy characteristics were collected during prenatal visits or at the time of admission to the hospital ward. Participants completed a questionnaire that collected information on their date and place of birth, history of pregestational diabetes and parity. All information provided was reviewed with the women by a doctor or midwife.

The HDI for each mother's country of origin was identified based on the UNDP human development report published for the year of delivery. The HDI index is the geometrical mean of the three dimension indices:

$$
\mathrm{HDI}=\left(\mathrm{I}_{\text {Health }} \cdot \mathrm{I}_{\text {Education }} \cdot \mathrm{I}_{\text {Income }}\right)^{1 / 3}
$$

Maximum and minimum values are set in order to transform the indicators expressed in different units into indices on a scale of 0 to 1 . These goalposts act as the "natural zeros" and "aspirational targets" from which component are standardized (Table 1).

These are examples of countries listed by HDI as included in the UNDP human development report in 2016:

Table $1 \mathrm{HDI}$ and its components

\begin{tabular}{llll}
\hline Dimension & Indicator & Minimum & Maximum \\
\hline Health & Life expectancy (years) & 20 & 85 \\
Education & $\begin{array}{l}\text { Expected years of } \\
\text { schooling (years) }\end{array}$ & 0 & 18 \\
& $\begin{array}{l}\text { Mean years of } \\
\text { schooling (years) }\end{array}$ & 0 & 15 \\
Standard of living & $\begin{array}{l}\text { Gross national income } \\
\text { per capita (2011 PPS\$) }\end{array}$ & 100 & 75,000 \\
\hline
\end{tabular}


Very High HDI (>0.80): Norway (0.949), Germany

(0.926), Spain (0.884), Croatia (0.827).

High HDI (0.70-0.80): Bulgaria (0.794), Cuba (0.775),

China (0.738), Uzbekistan (0.701).

Medium HDI (0.55-0.70): Moldova (0.699), Guatemala

(0.640), India (0.624), Zambia (0.579).

Low HDI (<0.55): Syria (0.536), Afghanistan (0.479),

Burundi (0.404), Niger (0.353).

Countries fall into four HDI categories. The first two groups are referred as developed countries and the last two are considered developing countries.

In order to simplify the analysis we created a group called "Medium-Low HDI" that contained the last two categories so we could differentiate patients that belonged to Very High, High and Medium-Low HDI of the maternal country of origin.

The outcome measures were perinatal/antenatal mortality, preeclampsia (PE), low birth weight (LBW), maternal mortality, gestational diabetes mellitus (GDM), preterm delivery (PTD) before 37 gestational weeks, PTD before 34 gestational weeks, abnormal cardiotocography (CTG) during delivery, C-section (CS) due to abnormal CTG, pH at birth $<7.10$, Apgar at $5 \mathrm{~min} \leq 7$, and resuscitation type $\geq 3$.

We excluded women with multiple pregnancies, miscarriages and foetal deaths before 22 weeks of gestation.

PE was diagnosed based on the guidelines of the International Society for the Study of Hypertension in Pregnancy; these guidelines define PE as the measurement of a systolic blood pressure of $140 \mathrm{mmHg}$ or greater and/ or a diastolic blood pressure of $90 \mathrm{mmHg}$ or greater on at least two occasions $4 \mathrm{~h}$ apart after 20 weeks of gestation. Proteinuria was defined as a urinary albumin-creatinine ratio greater than $300 \mathrm{mg}$ in a $24 \mathrm{~h}$ urine collection or two dipstick readings of ++ or higher.

GDM was diagnosed using a two-step approach. The O'Sullivan's test was performed on all women between 24 and 26 gestational weeks; this screening test involves measuring glucose levels one hour after the administration of $50 \mathrm{~g}$ oral glucose. The test results are considered positive when the glucose value is $140 \mathrm{mg} / \mathrm{dl}$ or higher. A $100 \mathrm{~g}$ oral glucose tolerance test was performed on those who had positive screening test results to establish the diagnosis of GDM.

PTD before 34 and 37 completed gestational weeks included pregnancies with spontaneous onset of labour with or without preterm rupture of membranes; however, those with iatrogenic delivery were excluded.

LBW neonates were defined as those with a birth weight below the 10th percentile for gestational age.

Based on standards set by the World Health Organization (WHO) and the National Centre for Health Statistics (NCHS) of the Centers for Disease Control and Prevention $(\mathrm{CDC})$, perinatal mortality and foetal mortality were defined as deaths occurring less than 7 days after birth and at a gestational age of 28 weeks or older, respectively [10].

The present study was approved by the Ethical Committee of Hospital General Universitario Gregorio Marañon de Madrid (Comité Ético de Investigacion Clínica, reference number OBS05042016).

\section{Statistical analysis}

We performed multivariate logistic regression analyses to estimate odds ratios (ORs) for the associations between maternal HDI and perinatal outcomes after adjustment for maternal age, pregestational diabetes history and parity. Maternal HDI served as a predictor variable and was divided in three categories: very high, high and medium/ low. The women were then divided into these three groups based on the HDI of their country of origin.

First, chi-square and Fisher's exact tests were used to identify significant between-group differences in categorical variables. Crude associations between maternal HDI and perinatal outcomes were calculated, and two-by-two multiple comparisons were performed with $p$-values adjusted using Holm's method.

The effect of maternal HDI on each perinatal outcome was explored using backward multivariate binary logistic regression.

Variable selection in the multivariate regression model was performed according to subject matter knowledge considered relevant predictors of perinatal outcome. Order of selection to evaluate the inclusion or exclusion of predictors was performed by descending statistical significance. Criteria to keep or retire predictors in the model took into account the clinical significance, that is, a variation of more or less than $10 \%$ of maternal HDI OR.

The odds ratios derived based on the results of the logistic regression and Wald test $p$-values were used to assess the statistical significance of the predictor variables within the models. The likelihood-ratio test was used to assess the fit of the model.

The significance level was set at $\alpha=0.05$, and all analyses were performed using SPSS 18 (SPSS Inc., Chicago, IL, USA) software.

\section{Results}

In total, 38,719 singleton infants who were born during the study period and for whom perinatal outcome data available were included in this study. The variables with the most missing values were $\mathrm{pH}$ at birth $(0.6 \%)$ and birth weight below the 10th percentile for gestational age $(0.3 \%)$.

Seventy percent of infants were born to patients from very high HDI countries; women from high HDI countries represented a $23 \%$ of the sample, and $7.1 \%$ of infants had mothers who originated from medium/low HDI countries. 
Information on the sample as parity, maternal age, pregestational diabetes (variables of adjustment in the model) for each HDI category are shown in Table 2 . The following four maternal deaths occurred after delivery in our centre: two cases of postpartum haemorrhage refractory to medical and surgical interventions, 1 case of chorioamnionitis at 26 weeks in a patient diagnosed with metastatic melanoma and 1 case of idiopathic intracranial hypertension with intracerebral haemorrhage and pneumonia after mechanical ventilation.

Comparisons between HDI groups and perinatal outcomes are presented in Table 3.

The initial crude associations and multiple comparisons between HDI groups and perinatal outcomes showed the following significant results, as seen in Table 4. In this analysis, we found that the rate of LBW was higher in the very high HDI group than the medium/low HDI group (OR 1.6, 95\% confidence interval $[\mathrm{CI}] 1.4-1.8)$. We did not identify statistically significant between-group differences in the rates of $\mathrm{PE}$, maternal mortality, neonatal-perinatal mortality or PTD before 34 weeks of gestation.

After adjusting for maternal age, pregestational diabetes history and parity (Table 5), the results of our multivariate model indicated that the rate of LBW was lower in the medium/low HDI group than the very high HDI group (aOR 0.63, 95\% CI 0.55-0.72). Additionally, the rate of PTD before 37 gestational weeks was higher in the medium/low HDI group than the very high HDI group (aOR 1.26, 95\% CI 1.04-1.53) and PE was identified significantly more frequently in women in the medium/low HDI group than women in the very high HDI group (aOR 1.35, 95\% CI 1.02-1.79). The proportion of women whose records indicated the occurrence of abnormal CTG during labour was higher in medium/ low HDI group than the very high HDI group (aOR $1.45,95 \%$ CI 1.23-1.70); accordingly, the rate of CS due to abnormal CTG was higher in the medium/low HDI group than very high HDI group (aOR 1.46, 95\% CI 1.23-1.73). Apgar 2 scores $\leq 7$ were more frequently identified in the medium/low HDI group than the very high HDI patients (aOR 1.45, 95\% CI 1.06-1.99), and resuscitation type $\geq 3$ was documented more frequently in

Table 2 Maternal age, parity and pregestational Diabetes for each HDI category

\begin{tabular}{lllll}
\hline & $\begin{array}{l}\text { Group A: } \\
\text { Very High HDI }\end{array}$ & $\begin{array}{l}\text { Group B: } \\
\text { High HDI }\end{array}$ & $\begin{array}{l}\text { Group C: } \\
\text { Medium-Low HDI }\end{array}$ & $\begin{array}{c}\text {-value } \\
\text { Global }\end{array}$ \\
\hline $\begin{array}{l}\text { Maternal Age } \\
\text { (IQR) }\end{array}$ & 33 & 31 & 31 & $<0.001$ \\
Parity (IQR) & $1(10-37)$ & $(26-35)$ & $(27-35)$ & \\
Pregestational & $132 / 26955$ & $1(1-2)$ & $2(1-2)$ & $<0.001$ \\
Diabetes n/N (\%) & $(0.49)$ & $18 / 8862$ & $7 / 2744$ & 0.001 \\
\hline
\end{tabular}

Table 3 Comparisons between HDI groups and perinatal outcomes

\begin{tabular}{lllll}
\hline & Group A: & Group B: & Group C: & p-value \\
& $\begin{array}{l}\text { Very } \\
\text { High HDI }\end{array}$ & High & $\begin{array}{l}\text { Medium-Low } \\
\text { HDI }\end{array}$ & Global \\
& HDI & \\
\hline Perinatal/antenatal death & $120(0.44)$ & $32(0.36)$ & $10(0.36)$ & 0.519 \\
Maternal death & $2(0.01)$ & $2(0.02)$ & $0(0.00)$ & 0.446 \\
Preeclampsia & $417(1.5)$ & $140(1.6)$ & $57(2.1)$ & 0.103 \\
LBW & $3862(14.3)$ & $844(9.5)$ & $261(9.5)$ & $<0.001$ \\
PTD <37 w & $959(3.5)$ & $386(4.3)$ & $122(4.4)$ & $<0.001$ \\
PTD <34 w & $332(1.2)$ & $128(1.4)$ & $37(1.3)$ & 0.280 \\
Abnormal CTG & $1247(4.6)$ & $467(5.3)$ & $180(6.6)$ & $<0.001$ \\
CS due to abnormal CTG & $1104(4.1)$ & $414(4.7)$ & $161(5.9)$ & $<0.001$ \\
Ph < 7.10 & $587(2.2)$ & $218(2.5)$ & $66(2.4)$ & 0.244 \\
Apgar 2 $\leq 7$ & $306(1.1)$ & $119(1.3)$ & $45(1.6)$ & 0.033 \\
Resuscitation $>3$ & $1783(6.6)$ & $660(7.5)$ & $205(7.5)$ & 0.010 \\
GDM & $768(2.8)$ & $199(2.2)$ & $81(2.9)$ & 0.008 \\
\hline
\end{tabular}

the records of high HDI patients than those of very high HDI patients (aOR 1.14, 95\% CI 1.04-1.25).

Statistically significant between-group differences were identified for maternal mortality neonatal-perinatal mortality, PTD before 34 weeks of gestation or $\mathrm{pH}$ at birth $<7.10$ in the final model.

\section{Discussion}

To our knowledge, this is the first study to investigate maternal HDI as the main social determinant of perinatal and pregnancy health. However, a recent study utilized maternal origin to define HDI scores and evaluate the extent to which stillbirths affected international comparisons of preterm delivery rates in low income countries [11].

This HDI simplifies and captures major social characteristics and encompasses various aspects of human development across countries in the form of a common score. Therefore, maternal origin can be categorized not only by race and ethnicity but also by income and educational level.

The aim of this paper was to identify differences in pregnancy outcomes between groups of patients demonstrating strong underlying inequalities that might interfere with antenatal care.

Our results demonstrate that after adjusting for potential confounding variables, a significant association between maternal HDI and a range of pregnancy complications still remained.

In summary, we can infer that patients with medium/ low HDI 1) did not have an excess risk of maternal or perinatal mortality; 2) had lower odds of LBW; 3) had greater odds of PTD before 37 weeks of gestation; 4) had greater odds of PE; and 4) had poorer neonatal 
Table 4 Significant multiple comparisons between HDI groups and perinatal outcomes

\begin{tabular}{lll}
\hline & $p$ & OR \\
\hline LBW & $<0.001$ & 1.6 \\
Group A: Very High HDI - Group B: High HDI & $<0.001$ & 1.6 \\
Group A: Very High HDI - Group C: Medium-Low HDI & $<0.001$ & 0.8 \\
PTD <37 W & $<0.001$ & 0.8 \\
Group A: Very High HDI - Group B: High HDI & 0.001 & $1.5-1.7$ \\
Group A: Very High HDI - Group C: Medium-Low HDI & 0.034 & 0.9 \\
Abnormal CTG & $<0.001$ & 0.7 \\
Group A: Very High HDI - Group B: High HDI & 0.020 & 0.8 \\
Group A: Very High HDI - Group C: Medium-Low HDI & $<0.001$ & $0.7-0.9$ \\
Group B: High HDI - Group C: Medium-Low HDI & 0.020 & $0.7-1.0$ \\
CS due to abnormal CTG & $<0.001$ & 0.9 \\
Group A: Very High HDI - Group B: High HDI & 0.024 & 0.7 \\
Group A: Very High HDI - Group C: Medium-Low HDI & $<0.001$ & $0.8-0.8$ \\
Group B: High HDI - Group C: Medium-Low HDI & 0.024 & $0.7-1.0$ \\
Resuscitation > 3 & 0.010 & $0.8-1.0$ \\
Group A: Very High HDI - Group B: High HDI & 0.018 & $0.6-0.8$ \\
GDM & 0.008 & $0.7-1.0$ \\
Group A: Very High HDI - Group B: High HDI & 0.009 & 0.9 \\
\hline
\end{tabular}

outcome, including a higher rates of abnormal CTG and CS due to abnormal CTG and lower Apgar scores at 5 min.

A key finding of this study was that belonging to the lower HDI group was associated with lower odds of LBW when compared with women from MEDC. This finding confirms the results of previous studies wherein the risk of poor prenatal outcome was greater among native-born than immigrant mothers [12]. Several factors may have contributed to the better pregnancy outcomes identified in this group, and especially newborn weight. Immigrants tend to engage in healthier behaviours, such as consuming less alcohol and cigarettes and having healthier diets and stronger family ties. Another possible explanation for this finding is aligned with the "healthy migrant effect," which suggests that a minimum level of health is required to migrate; thus, only a segment of the healthy population will migrate to the host country [13].

Another study evaluated areas with high levels of social deprivation, as defined by an index based on different factors such as the presence of universal health insurance, educational level, occupational status, household income or living with a partner, and identified results that were different from those of our study. In that analysis, the adjusted model indicated that their index was associated with late prenatal care (OR 5.8, 95\% CI 4.6 to 7.2 ) and small for gestational age (OR $1.5,95 \%$ CI 1.1 to 1.9 ) [14].
As previously described, we also found that patients originating from medium/low HDI countries had a higher probability of spontaneous PTD before 37 weeks of gestation relative to their higher HDI counterparts. Some authors have suggested that a lower level of maternal education may be an underlying cause of the increased early delivery risk observed in less educated populations. These patients may be poorer and have other comorbidities, both of which have been identified as independent risk factors for preterm birth. These patients may also be less involved in decision making and planning during pregnancy, and a previous study suggested that a greater proportion of these women believed that delivery at 34 gestational weeks was safe $[15,16]$. A population study including more than 500,000 singleton pregnancies in the UK reported Afro-Caribbean patients to be at 1.6 times greater odds of PTD before 37 gestational weeks than Caucasian patients [17].

Other authors using social deprivation variables and other poverty indicators have not identified an association between these factors and preterm birth [18].

The manner in which maternal origin and ethnicity are associated with PE remains poorly understood. Without knowledge of the underlying causes of disease, we can only explore risk factors. A retrospective cohort study including 127,544 low risk women in the USA examined the role of maternal ethnicity in prenatal outcomes using multivariate models adjusted for various confounding variables, and the rates of $\mathrm{PE}$ were higher 
Table 5 Multivariate logistic regression adjusted for maternal age, pregestational diabetes and parity

\begin{tabular}{|c|c|c|c|c|}
\hline & $\mathrm{N}$ & $p$ & $\mathrm{aOR}$ & $\mathrm{Cl} 95 \%$ \\
\hline Perinatal/antenatal death & 38,718 & 0.51 & & \\
\hline Global Maternal HDI & & 0.52 & & \\
\hline High HDI - Very High HDI & & 0.298 & 0.81 & $0.55-1.20$ \\
\hline Medium-Low HDI - Very High HDI & & 0.547 & 0.82 & $0.43-1.57$ \\
\hline Maternal death & 38,718 & 0.412 & & \\
\hline Global Maternal HDI & & 0.537 & & \\
\hline High HDI - Very High HDI & & 0.265 & 3.05 & $0.43-21.66$ \\
\hline Medium-Low HDI - Very High HDI & & 0.988 & 0 & $0.00-$ \\
\hline Preeclampsia & 38,718 & 0.124 & & \\
\hline Global Maternal HDI & & 0.105 & & \\
\hline High HDI - Very High HDI & & 0.806 & 1.02 & $0.85-1.24$ \\
\hline Medium-Low HDI - Very High HDI & & 0.034 & 1.35 & $1.02-1.79$ \\
\hline LBW & 38,535 & $<0.001$ & & \\
\hline Global Maternal HDI & & $<0.001$ & & \\
\hline High HDI - Very High HDI & & $<0.001$ & 0.63 & $0.58-0.68$ \\
\hline Medium-Low HDI - Very High HDI & & $<0.001$ & 0.63 & $0.55-0.72$ \\
\hline PTD $<37$ weeks & 38,718 & 0.001 & & \\
\hline Global Maternal HDI & & $<0.001$ & & \\
\hline High HDI - Very High HDI & & 0.001 & 1.24 & $1.10-1.40$ \\
\hline Medium-Low HDI - Very High HDI & & 0.017 & 1.26 & $1.04-1.53$ \\
\hline PTD $<34$ weeks & 38,718 & 0.288 & & \\
\hline Global Maternal HDI & & 0.281 & & \\
\hline High HDI - Very High HDI & & 0.117 & 1.18 & $0.96-1.45$ \\
\hline Medium-Low HDI - Very High HDI & & 0.59 & 1.1 & $0.78-1.55$ \\
\hline Abnormal CTG & 38,582 & $<0.001$ & & \\
\hline Global Maternal HDI & & $<0.001$ & & \\
\hline High HDI - Very High HDI & & 0.012 & 1.15 & $1.03-1.28$ \\
\hline Medium-Low HDI - Very High HDI & & $<0.001$ & 1.45 & $1.23-1.70$ \\
\hline CS due to abnormal CTG & 38,590 & $<0.001$ & & \\
\hline Global Maternal HDI & & $<0.001$ & & \\
\hline High HDI - Very High HDI & & 0.018 & 1.15 & $1.03-1.29$ \\
\hline Medium-Low HDI - Very High HDI & & $<0.001$ & 1.46 & $1.23-1.73$ \\
\hline $\mathrm{pH}<7.10$ & 38,474 & 0.25 & & \\
\hline Global Maternal HDI & & 0.245 & & \\
\hline High HDI - Very High HDI & & 0.112 & 1.14 & $0.97-1.33$ \\
\hline Medium-Low HDI - Very High HDI & & 0.87 & 1.11 & $0.86-1.44$ \\
\hline Apgar $2 \leq 7$ & 38,590 & 0.04 & & \\
\hline Global Maternal HDI & & 0.034 & & \\
\hline High HDI - Very High HDI & & 0.113 & 1.19 & $0.96-1.47$ \\
\hline Medium-Low HDI - Very High HDI & & 0.02 & 1.45 & $1.06-1.99$ \\
\hline
\end{tabular}

Table 5 Multivariate logistic regression adjusted for maternal age, pregestational diabetes and parity (Continued)

\begin{tabular}{lllll}
\hline Resuscitation $>3$ & 38,590 & 0.011 & & \\
Global Maternal HDI & 0.01 & & \\
High HDI - Very High HDI & 0.006 & 1.14 & $1.04-1.25$ \\
Medium-Low HDI - Very High HDI & 0.084 & 1.14 & $0.98-1.33$ \\
GDM & 38,717 & 0.007 & & \\
Global Maternal HDI & 0.008 & & \\
High HDI - Very High HDI & 0.003 & 0.79 & $0.67-0.92$ \\
Medium-Low HDI - Very High HDI & 0.743 & 0.8 & $0.82-1.31$ \\
\hline
\end{tabular}

among African American than white women (OR 1.49, CI 95\% 1.33-1,72) [19]. Maternal origin may also be associated with hypertensive disorders in pregnancy due to its link with socioeconomic status, which has demonstrated an inverse relationship with PE development.

In our centre, immigrant women had greater odds of poor neonatal outcomes, including abnormal CTG results, CS due to abnormal CTG results and Apgar scores at $5 \mathrm{~min}$. A large study conducted in Canada that included more than 800,000 births showed that the rate of neonatal ICU admissions was higher among infants born to immigrants from South Asia, Africa and the Caribbean than the infants of native Canadian mothers (RR 1.41 CI 95\%, 1.36 to 1.46) [20]. Newborns admitted to the ICU may be at increased risk of neonatal mortality and have a significantly higher probability of longterm morbidity [21].

Country of origin has been used to identify maternal and perinatal outcomes; in some regions, the relative risk of neonatal ICU admission has been found to differ between mothers and newborns [22].

\section{Strengths and limitations}

The main strengths of our study are its large sample size and the low rate of missing perinatal outcome data. Our findings regarding the association between maternal origin and pregnancy results are consistent with the results of prior studies.

However, our research also has several limitations. First, we did not consider other maternal and pregnancy characteristics that may be relevant confounders such as maternal smoking, body mass index or method of conception.

Assessments of a mother's social situation and level of poverty should include educational achievement, maternal and family income, Spanish language acquisition and familial support level. We attempted to divide the patients into groups in a simple manner that was based on maternal HDI; additionally, we obtained as much information as we could regarding the mothers' social situation, as indicated by their country of origin. 
We also lacked information about cultural and health behaviours, other maternal medical conditions such as chronic hypertension and rheumatological diseases, and antenatal care, which precluded us from assessing their impact on pregnancy outcomes. Among immigrants, adverse birth outcomes have been found to be associated with inadequate obstetric care due to difficulties accessing medical services [23].

We used the Strengthening the Reporting of Observational Studies in Epidemiology (STROBE) statement checklist as an instrument to evaluate the quality of our work and assure the presence of items that should be included in reports of observational studies [24].

\section{Conclusions}

In conclusion, this study contributes to the body of literature by generating a better understanding of the manner in which maternal HDI may serve as a score that integrates crucial social factors related to pregnancy and perinatal outcome in our country. We identified lower odds of LBW among immigrants from lower HDI countries despite the identification of greater odds of PTD before 37 gestational weeks, PE and poorer neonatal outcomes in this group.

These findings emphasize the importance of assessing maternal socioeconomic status to facilitate an understanding of the relationship between migration and perinatal outcome. Future studies should continue to evaluate the impact of political and economic interventions on pregnancy outcomes in socially deprived populations.

Further research should also be conducted to more accurately assess the socioeconomic characteristics of immigrant populations and their association with obstetrical outcomes. Generating increased knowledge of these characteristics and their associations requires the use of tools that can provide information regarding adherence to pregnancy follow up, programmes, access to prenatal care, and other indicators linked to the use of medical services.

\section{Abbreviations}

CDC: Centers for Disease Control and Prevention; CS: C-Section CTG: Cardiotocography; GDM: Gestational diabetes mellitus; GNI: Gross national income per capita; HDI: Human Development Index; ICU: Intensive care unit; MEDC: More economically developed countries; NCHS: National Centre for Health Statistics; PE: Preeclampsia; PTD: Preterm delivery; UN: United Nations; UNDP: United Nations Development Programme; WHO: World Health Organization

\section{Acknowledgements}

Not applicable.

\section{Funding}

No funding was received for this study.

\section{Availability of data and materials}

All data supporting the findings of this study can be made available upon request.

\section{Authors' contributions}

GTLS designed and carried out the study, performed literature review and drafted the manuscript. ASJ performed the statistical analysis and prepared the final manuscript. DVA, PRMP, CHI, SPA, LBS and DLLJ revised the final manuscript. All authors read and approved the final manuscript.

\section{Ethics approval and consent to participate}

This study was ethically approved by the Ethical Committee of Hospital General Universitario Gregorio Marañon de Madrid (Comité Ético de Investigacion Clínica, reference number OBS05042016). This committee declared there is no need to obtain informed consent to participate from patients for this retrospective study (Ref number OBS05042016).

\section{Consent for publication}

Not applicable.

\section{Competing interests}

The authors declare that they have no competing interests.

\section{Publisher's Note}

Springer Nature remains neutral with regard to jurisdictional claims in published maps and institutional affiliations.

\section{Author details}

'Department of Obstetrics and Gynaecology, Hospital General Universitario Gregorio Marañón, Universidad Complutense de Madrid, 28029 Madrid, ES, Spain. ${ }^{2}$ Department of Internal Medicine, Hospital Universitario, Principe de Asturias de Alcalá de Henares, Madrid, Spain.

Received: 8 May 2017 Accepted: 18 September 2017

Published online: 21 September 2017

\section{References}

1. Luque-Fernandez MA, Franco M, Gelaye B, Schomaker M, Garitano IG, D'Este $C$, et al. Unemployment and stillbirth risk among foreign-born and Spanish pregnant women in Spain, 2007-2010: a multilevel analysis study. Eur J Epidemiol. 2013;28:991-9. https://doi.org/10.1007/s10654-013-9859-y.

2. Larrañaga I, Santa-Marina L, Begiristain H, Machón M, Vrijheid M, Casas M, et al. Socio-economic inequalities in health, habits and selfcare during pregnancy in Spain. Matern Child Health J. 2012;17:1315-24.

3. Bonet M, Smith LK, Pilkington H, Draper ES, Zeitlin J. Neighbourhood deprivation and very preterm birth in an English and French cohort. BMC Pregnancy Childbirth. 2013:13:97. https://doi.org/10.1186/1471-2393-13-97.

4. Carstairs V, Morris R. Deprivation: explaining differences in mortality between Scotland and England and Wales. BMJ. 1989;299:886-9. https://doi. org/10.1136/bmj.299.6704.886.

5. Salmond C, Crampton P, King P, Waldegrave C. NZiDep: a New Zealand index of socioeconomic deprivation for individuals. Soc Sci Med. 2006;62: 1474-85. https://doi.org/10.1016/j.socscimed.2005.08.008.

6. Acevedo-Garcia D, Soobader MJ, Berkman LF. The differential effect of foreign-born status on low birth weight by race/ethnicity and education. Pediatrics. 2005;115:e20-30. https://doi.org/10.1542/peds.2004-1306.

7. Hessol NA, Fuentes-Afflick E. The perinatal advantage of Mexican-origin Latina women. Ann Epidemiol. 2000;10:516-23. https://doi.org/10.1016/ S1047-2797(00)00073-9.

8. United Nations Development Programme (UNDP). Summary human development report 2013. http://hdr.undp.org/sites/default/files/reports/14/ hdr2013 en complete.pdf. Accessed 10 April 2013.

9. United Nations Development Programme (UNDP). Reports (1990-2013) Human development reports (HDR). http://hdr.undp.org/sites/default/files/ reports/14/hdr2013_en_complete.pdf. Retrieved 22 Sept 2013.

10. Barfield WD. Standard terminology for fetal, infant, and perinatal deaths. Pediatrics. 2016;137:e20160551. https://doi.org/10.1542/peds.2016-0551.

11. Morisaki N, Ganchimeg T, Vogel JP, Zeitlin J, Cecatti JG, Souza JP, et al. Impact of stillbirths on international comparisons of preterm birth rates: a secondary analysis of the WHO multi-country survey of maternal and Newborn health. BJOG. 2017; https://doi.org/10.1111/1471-0528.14548.

12. Moore S, Daniel M, Auger N. Socioeconomic disparities in low birth weight outcomes according to maternal birthplace in Quebec, Canada. Ethn Health. 2009;14:61-74. https://doi.org/10.1080/13557850802071132. 
13. Racape J, Schoenborn C, Sow M, Alexander S, De Spiegelaere M. Are all immigrant mothers really at risk of low birth weight and perinatal mortality? The crucial role of socio-economic status. BMC Pregnancy Childbirth. 2016; 16:75. https://doi.org/10.1186/s12884-016-0860-9.

14. Opatowski M, Blondel B, Khoshnood B, Saurel-Cubizolles MJ. New index of social deprivation during pregnancy: results from a national study in France. BMJ Open. 2016;6:e009511. https://doi.org/10.1136/bmjopen-2015-009511.

15. Goldenberg RL, McClure EM, Bhattacharya A, Groat TD, Stahl PJ. Women's perceptions regarding the safety of births at various gestational ages. Obstet Gynecol. 2009;114:1254-8. https://doi.org/10.1097/AOG. 0b013e3181c2d6a0.

16. Auger $N$, Leduc L, Naimi Al, Fraser WD. Delivery at term: impact of university education by week of gestation. J Obstet Gynaecol Can. 2016;38:118-24. https://doi.org/10.1016/j.jogc.2015.11.001.

17. Balchin I, Steer PJ. Race, prematurity and immaturity. Early Hum Dev. 2007; 83:749-54. https://doi.org/10.1016/j.earlhumdev.2007.09.003

18. Poeran J, Maas AFG, Birnie E, Denktas S, Steegers EAP, Bonsel GJ. Social deprivation and adverse perinatal outcomes among Western and nonWestern pregnant women in a Dutch urban population. Soc Sci Med. 2013; 83:42-9. https://doi.org/10.1016/j.socscimed.2013.02.008.

19. Caughey AB, Stotland NE, Washington AE, Escobar GJ. Maternal ethnicity, paternal ethnicity, and parental ethnic discordance: predictors of preeclampsia. Obstet Gynecol. 2005;106:156-61. https://doi.org/10.1097/01. AOG.0000164478.91731.06

20. Admission ICU, Medcalf KE, Park AL, Vermeulen MJ, Ray JG. Maternal origin and risk of neonatal and maternal. Crit Care Med. 2016:44:1314-26.

21. Kirkby S, Greenspan JS, Kornhauser M, Schneiderman R. Clinical outcomes and cost of the moderately preterm infant. Adv Neonatal Care. 2007;7:80-7. https://doi.org/10.1097/01.ANC.0000267913.58726.f3.

22. Wahlberg A, Rööst M, Haglund B. Increased risk of severe maternal morbidity (near-miss) among immigrant women in Sweden: a population register-based study. BJOG. 2013;120:1605-11. discussion 1612

23. Essén B, Bödker B, Sjöberg NO, Langhoff-Roos J, Greisen G, Gudmundsson S, et al. Are some perinatal deaths in immigrant groups linked to suboptimal perinatal care services? BJOG. 2002;109:677-82.

24. von Elm E, Altman DG, Egger M, Pocock SJ, Gøtzsche PC, Vandenbroucke JP, et al. The Strengthening the Reporting of Observational Studies in Epidemiology (STROBE) statement: guidelines for reporting observational studies. J Clin Epidemiol. 2008;61:344-9. https://doi.org/10.1016/j.jclinepi.2007.11.008.

\section{Submit your next manuscript to BioMed Central and we will help you at every step:}

- We accept pre-submission inquiries

- Our selector tool helps you to find the most relevant journal

- We provide round the clock customer support

- Convenient online submission

- Thorough peer review

- Inclusion in PubMed and all major indexing services

- Maximum visibility for your research

Submit your manuscript at www.biomedcentral.com/submit

CBiomed Central 